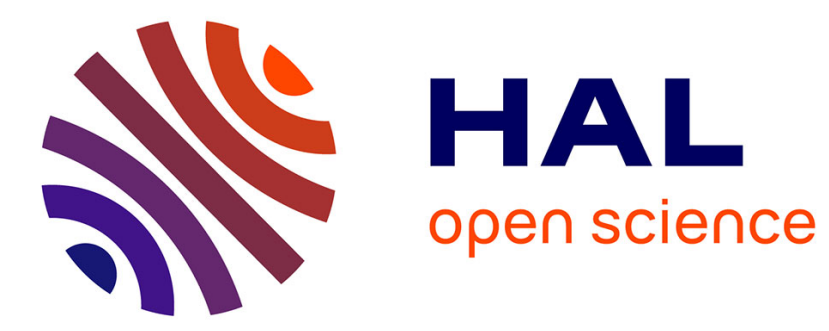

\title{
Aqueous foams stabilized solely by particles
}

Antonio Stocco, Emmanuelle Rio, Bernard P. Binks, Dominique Langevin

\section{To cite this version:}

Antonio Stocco, Emmanuelle Rio, Bernard P. Binks, Dominique Langevin. Aqueous foams stabilized solely by particles. Soft Matter, 2011, 7 (4), pp.1260-1267. hal-00788618

\section{HAL Id: hal-00788618 https://hal.science/hal-00788618}

Submitted on 15 Feb 2013

HAL is a multi-disciplinary open access archive for the deposit and dissemination of scientific research documents, whether they are published or not. The documents may come from teaching and research institutions in France or abroad, or from public or private research centers.
L'archive ouverte pluridisciplinaire HAL, est destinée au dépôt et à la diffusion de documents scientifiques de niveau recherche, publiés ou non, émanant des établissements d'enseignement et de recherche français ou étrangers, des laboratoires publics ou privés. 


\title{
Aqueous foams stabilized solely by particles
}

\author{
A. Stocco ${ }^{1 \#}$, E. Rio ${ }^{1}$, B.P. Binks ${ }^{2}$ and D. Langevin ${ }^{1 *}$ \\ ${ }^{1}$ Laboratoire de Physique des Solides, Université Paris-Sud, UMR CNRS 8502, \\ Bâtiment 510, 91405 Orsay cedex, France. \\ \#present address: Max Planck Institute of Colloids and Interfaces, 14476 Golm - Potsdam, \\ Germany. \\ ${ }^{2}$ Surfactant \& Colloid Group, Department of Chemistry, University of Hull, \\ Hull, HU6 7RX, UK.
}

\begin{abstract}
Foams are dispersions of bubbles in liquids, often water. They are frequently stabilized by surfactant or polymer, but like Pickering emulsions, they can be also stabilized solely by particles. If the particles have a moderate hydrophobicity, the foams can be extremely stable (lifetimes of the order of years). Due to technical preparation difficulties, very few studies can be found in the literature to date. We will discuss the origin of these difficulties. We will also describe experiments using partially hydrophobic fumed silica particles, in which the foam properties were correlated with the properties of spread and adsorbed layers of these particles at the air-water interface. These combined experiments allowed us to define the conditions necessary to stop bubble disproportionation.
\end{abstract}

\section{Introduction}

Pickering emulsions ${ }^{1}$, i.e. emulsions stabilized solely by solid particles of nano- or micrometer size, can be remarkably stable. It has been shown recently that colloidal particles of controlled hydrophobicity can significantly enhance foam stability ${ }^{2-4}$, a matter of great technological and commercial significance. An example of application concerns metallic foams, where oxide particles can play the role of stabilizers. ${ }^{5}$ Note that foams have already been extensively used in applications such as flotation of mineral particles in which the bubble surfaces are covered by particles. ${ }^{6}$ The fact that hydrophobic particles are currently used as antifoams ${ }^{7}$ points out the importance of the nature of the particle surface in foam stabilisation/destabilisation. It has been also shown recently that single bubbles ${ }^{8}$ stabilized by certain particles are similarly extremely stable. The existing studies support the picture of air-liquid interfaces covered by particle layers forming a colloidal armour ${ }^{9,10}$ which inhibits the two main ageing mechanisms of the foams: bubble coalescence (film rupture) and coarsening or disproportionation (exchange of gas between bubbles due to differences in Laplace pressure). This may lead to foam lifetimes of months and above. ${ }^{11}$ Recent reviews can be consulted for more details. ${ }^{12-14}$ 
In most cases, the desired particle hydrophobicity is obtained by mixing hydrophilic particles and surfactants, which makes difficult the interpretation of measurements: the particle surface coverage can be inhomogeneous and/or vary with time if surfactant diffuses on the surface and/or away from it. Furthermore, existing experiments on foams were rarely associated to characterisation of particle surface layers. In this paper, we will summarize the work done on aqueous foams stabilized by partially hydrophobic fumed silica nanoparticles, where independent studies of particle surface layers allow a better understanding of the extreme foam stability. In these studies, a clear maximum in foamability was found for particles of intermediate hydrophobicity and such foams were stable indefinitely. ${ }^{3}$ Other foam studies were carried out and include multiple light scattering and X-ray tomography investigations. ${ }^{11,15,16}$ In addition to foam studies, the properties of the particle layers at the air-water interface have been investigated by various means: static and dynamic surface tension, surface rheology, ellipsometry and Brewster angle imaging. ${ }^{16-20}$

\section{Materials}

Particles. Fumed silica nanoparticles were chemically coated with a short-chain silane reagent (dichlorodimethylsilane) by the manufacturer (Wacker-Chemie, Germany). The hydrophobic character of the particles is expressed in terms of the percentage of surface silanol groups SiOH. The maximum in foamability for aqueous systems corresponds to a residual percentage of around $35 \%$ $\mathrm{SiOH}^{3}{ }^{3}$ The primary particles are quasi-spherical, of $20-30 \mathrm{~nm}$ in diameter, and are aggregated during the manufacturing process into clusters of about $200 \mathrm{~nm}$ in diameter ${ }^{21}$. In the following, we will discuss the results obtained with particles having about $35 \%$ surface silanol groups unless stated otherwise.

Particle dispersions. Aqueous dispersions of particles were prepared using Milli-Q water and a small amount of ethanol ( $<2 \mathrm{wt}$. \%) to facilitate particle wetting. The subsequent sonication ( one hour) turned out to be a fundamental step to ensure reproducibility of the measurements. The highest particle concentration that could be achieved in this way was $1 \mathrm{wt} . \%$. The dispersions were generally stable for up to a month.

In order to determine the aggregate size after sonication, standard light scattering studies were conducted and confirmed that the preparation protocol described above led reproducibly to fairly monodisperse aggregates. ${ }^{16}$ Static measurements led to a radius of gyration of the aggregates of $R_{g}=$ $71 \pm 4 \mathrm{~nm}$ and a positive second virial coefficient, corresponding to repulsive particle interactions. Dynamic measurements led to a hydrodynamic radius $R_{h}=85 \pm 10 \mathrm{~nm}$ and a polydispersity index $\sigma=$ $\left(\left\langle R^{2}\right\rangle-\langle R\rangle^{2}\right) /\langle R\rangle^{2} \sim 0.2$. Thus, the particle aggregates are fairly monodisperse. The ratio $R_{h} / R_{g}$ is about 1.2 , close to $(5 / 3)^{0.5}$, indicating that the aggregates have approximately spherical shapes. Note that electron microscopy studies suggest that the aggregates are rather polydisperse and ramified, but this is perhaps due to aggregation during sample drying.

The repulsive interactions are most likely partly due to electrostatic repulsion coming from dissociated $\mathrm{SiOH}$ groups in water. At the pH of the dispersions ( 5.5), the surface potential $\psi$ of silica is about $-100 \mathrm{mV}^{22}$ If only $35 \%$ of $\mathrm{SiOH}$ groups remain after surface modification, one expects that the surface potential will be increased up to $-35 \mathrm{mV}$. The ionic concentration $\mathrm{c}_{\text {ion }}$ is linked to the surface charge density $\sigma$ by the Grahame equation: 


$$
\sigma=\left(8 \mathrm{k}_{\mathrm{B}} \mathrm{T} \mathcal{N} \mathrm{c}_{\text {ion }} \varepsilon\right)^{1 / 2} \sinh \left(\mathrm{e} \psi / 2 \mathrm{k}_{\mathrm{B}} \mathrm{T}\right)
$$

where $\mathrm{k}_{\mathrm{B}}$ is the Boltzmann constant, $\mathrm{T}$ the absolute temperature, $\mathcal{N}$ the Avogadro number, $\varepsilon$ the dielectric constant of the solvent, e the electron charge and $\psi$ the surface potential. The ionic concentration is also related to the charge density $\sigma$ and to the actual bulk particle concentration $c$ by:

$$
\mathrm{c}_{\text {ion }}=3 \mathrm{c} \sigma /(\mathcal{N} R \text { e d })
$$

where $d$ is the density of the particles relative to water $(d \sim 2)$ and $R$ is their radius. This leads to $c_{\text {ion }} \sim$ $10^{-4} \mathrm{M}$ for $\mathrm{c}=0.1 \mathrm{wt} . \%$. This ionic concentration corresponds to a Debye electrostatic screening length of $30 \mathrm{~nm}$, comparable to the primary particle size. For $\mathrm{c}=1 \mathrm{wt} . \%$, the screening length is smaller, about $3 \mathrm{~nm}\left(\mathrm{c}_{\text {ion }} \sim 10 \mathrm{mM}\right)$.

It should be mentioned that additional short range steric repulsions due to hydration layers on particle surfaces may be present, as for unmodified silica nanoparticles. ${ }^{23}$

\section{Results and Discussion}

We will first recall the interfacial studies of different types of particle layers, either spread at the airwater interface or adsorbed from particle dispersions. We will then show the correlations between these results and foam properties.

\subsection{Surface properties}

\subsubsection{Spread particle layers}

Spread layers were made using particle dispersions in iso-propyl alcohol. In these experiments, the dispersions were spread onto the surface of water contained in a Langmuir trough equipped with teflon barriers. The surface concentration of particles $\Gamma$ was varied either by compressing the layer with the barriers or by adding, step by step, small quantities of particle dispersion (successive deposition). We will use the notion of surface pressure $\Pi$, equal to the difference between the surface tension of pure water $\gamma_{w}$ and the surface tension of water covered by particles $\gamma$. $\Pi$ varies with particle surface concentration $\Gamma$, and a typical curve is shown in Figure 1(a). The surface pressure is zero at low surface concentration and increases rather abruptly above $\Gamma=30 \mathrm{mg} / \mathrm{m}^{2}$. Brewster Angle Microscopy (BAM) images are also shown in Figure 1(b): in regions of zero surface pressure (left), the layer is made of disconnected islands; at the onset of the surface pressure increase (center), the layer is uniform and when the surface pressure changes slope around 50 $\mathrm{mg} / \mathrm{m}^{2}$ (right), the layer starts to buckle. Similar buckling effects were seen with the same particles at oil-water interfaces ${ }^{24}$ and with micron-sized particles at air-water interfaces. ${ }^{25,26}$

Ellipsometry allowed the determination of the layer thickness I and of the surface coverage $\phi_{s}$ (area covered by the particles divided by the total area). ${ }^{18}$ It was found that the layer thickness is comparable to the diameter $2 \mathrm{R}$ of the particles aggregates, i.e. around $170 \mathrm{~nm}$. This allows us to estimate the surface coverage as $\phi_{s}=3 \Gamma /(4 \rho R)$. It was found that the surface coverage $\phi_{s}$ is low, around 0.2 , at the onset of the surface pressure increase $\left(\Gamma \sim 30 \mathrm{mg} / \mathrm{m}^{2}\right)$, suggesting the existence of 
long range repulsive forces, probably of electrostatic origin. For nanoparticles at a liquid-air surface, electrostatic interactions are of the Debye-Hückel type instead of forces algebraically decaying with distance for micron-sized particles. ${ }^{27,}{ }^{28}$ Even if van der Waals forces hold the primary silica particles together in the clusters, these clusters cannot approach to small distances due to the repulsive forces. Note that the clusters are rough and it can be shown that the attractive capillary interaction between them at these distances is negligible. ${ }^{29}$ If the surface potential of the particles is as in bulk, a combination of Debye-Hückel repulsion and van der Waals attraction results in a shallow minimum in the overall interaction potential occurring at distances between particles close to the Debye length. A value of $\phi_{\mathrm{s}}$ of 0.2 corresponds to a distance between particles of about twice their diameter, i.e. according to the above, to a Debye length of about $100 \mathrm{~nm}$. The corresponding ionic concentration is about $10^{-5} \mathrm{M}$, close to that expected for water at the natural $\mathrm{pH}$.

Figure 1 also reveals non-equilibrium features: the surface pressure depends on the velocity of compression and on the orientation of the Wilhelmy plates used to measure the surface tension. The observation of a difference between the surface pressures measured with the two plates, that decreases when the velocity of the barriers decreases, is characteristic of solid-like layers with long relaxation times. A similar behavior was found for micron-sized particle layers. ${ }^{26}$ As layers of micronsized particles, ${ }^{30}$ the layers discussed here possess a finite shear modulus that vanishes above a strain typically of the order of a few percent. ${ }^{19}$ When the strain is diminished, the layer heals, i.e. the shear modulus increases and retrieves its initial value after a long time (a few hours). Independent BAM observations reveal that the layers can be fractured under expansion or shear and heal after a similar long time, suggesting that the behaviour of the shear modulus is associated to healing of defects in the layers. A layer compressed above $50 \mathrm{mg} / \mathrm{m}^{2}$ buckles, but buckling disappears after a few hours and the layer becomes homogeneous in thickness as in Figure 1 (b) (center).

Figure 1(a) also shows that layers obtained by compression of an initially dilute layer are more compact than those obtained by successive depositions, and that their elastic compression moduli are larger. This is in line with the ellipsometry investigations as discussed above. When the compressed layers are decompressed, hysteresis is observed. ${ }^{17}$ The layer is apparently compacted further and the surface coverage increases. Further compression-expansion cycles do not lead to further changes in surface pressure.

In summary, these nano- and micron-sized particle layers present many similarities with granular media (in two dimensions), namely pronounced non-equilibrium features and time history dependencies associated with consolidation events. In contrast to micron-sized particles, however, long range repulsion is present that prevents particles from coming into contact and solid friction is absent. This is probably why fractures heal reversibly. The deposited nanoparticle layers appear softer than the compressed ones, probably because their formation does not favor consolidation. We will see in the following that the surface pressures and viscoelastic properties of deposited layers are close to those of adsorbed layers.

\subsubsection{Adsorbed particle layers}

Contrary to surfactant molecules, solid particles can adsorb irreversibly at the interface, with adsorption energies being of the order of several thousands of $k_{B} T$. Thus, it is very difficult to force adsorbed particles out of the interface. The adsorption energy $W_{r}$ of a single particle is related to the 
contact angle $\theta$ that the particle makes with the interface, the air-water surface tension $\gamma_{w}$, and the radius of the particles $R$ by

$$
W_{\mathrm{r}}=\gamma_{w} R^{2} \pi(1 \pm \cos \theta)^{2}
$$

in which the sign within the brackets corresponds to particle centers above (+) or below (-) the interface. For particles with $35 \% \mathrm{SiOH}$ surface groups and a contact angle at the air-water interface of $120^{\circ}{ }^{31}$ one estimates $W_{r}$ to be $1400 k_{B} T$ for the primary silica particles (radius $\sim 10 \mathrm{~nm}$ ).

Adsorption at the free surface of aqueous particle dispersions was monitored by ellipsometry. ${ }^{16}$ Even after one day, the ellipsometric signal was the same for pure water and for the particle dispersions in the whole concentration range between 0.1 and $0.7 \mathrm{wt} . \%$. This implies that no significant amount of particles was adsorbed at the interface. Using BAM, islands of particles floating at the water surface were observed, but their area fraction was not high enough to contribute to the ellipsometric signal. These results are in apparent contradiction with those obtained using spread particle monolayers. The main difference between the two cases is that in the latter all particles are placed directly at the surface where they remain due to large desorption energies. In the case of aqueous dispersions, however, the particles need to overcome two obstacles: firstly, they need to diffuse against gravity from the bulk to the surface (silica particles are significantly heavier than water with a density $\rho \sim 2 \mathrm{~g}$ $\mathrm{cm}^{-3}$ ), and secondly, they need to overcome interfacial energy barriers if present.

In order to enhance particle adsorption, strong sonication (for few minutes) was applied to the dispersions. Performing ellipsometric scans immediately after sonication reveals significant changes of the interfacial profile, i.e. significant particle adsorption, the signal becoming similar to that obtained with spread layers. At sufficiently high particle concentrations, a completely different scenario is observed: a network-like structure of particles appears and does not change within 24 hours. ${ }^{16}$ It may seem strange to observe the formation of networks, knowing that the particle interactions are repulsive. We can however hypothesize that the energy supplied by sonication, allowing the particles to overcome the adsorption barrier and adsorb at the surface, also allows some of these particles to overcome the repulsive barrier between themselves (which has the same origin). Sonication may also produce air bubbles in the water phase, to which particles could be attached and be brought to the surface as in flotation.

Further investigations of the adsorbed layers were performed using the pendant drop/rising bubble method. The surface tension variation with time $t$ (dynamic surface tension) for a range of particle concentrations was studied. In the case of the most concentrated dispersions, the surface tension remains constant with time, but a very slow evolution is seen with the less concentrated dispersions. The evolution is too slow to correspond to diffusion-controlled adsorption. Indeed, the mean distance between particles and the surface is about $\Gamma / c$, i.e. of the order of $1 \mu \mathrm{m}$ for $c=0.3 \mathrm{wt} . \%$ (taking $\Gamma=50 \mathrm{mg} / \mathrm{m}^{2}$ ). The mean square displacement for a diffusing particle is given by $\mathrm{L}^{2}=2 \mathrm{Dt}$, with $D=2.5 \times 10^{-12} \mathrm{~m}^{2} \mathrm{~s}^{-1}$, as evaluated by dynamic light scattering. ${ }^{16}$ Taking $\mathrm{L}=1 \mu \mathrm{m}$ gives a time $\mathrm{t}<$ $0.2 \mathrm{~s}$, i.e. much shorter than for the long time evolution of the surface tension. This evolution is therefore likely due to the adsorption barrier. Once a few particles are adsorbed at the surface, the surface becomes charged and repels the new particles approaching the surface. Similar electrostatic barriers were observed in the case of surfactant molecules: molecules accumulate in the sub-surface region until they can overcome the barrier after a certain time. ${ }^{32}$ Oscillating bubble experiments confirmed the existence of an adsorption barrier: the surface tension values obtained for a static 
bubble after long waiting times can be reached much more rapidly by oscillating the bubble and hence by providing an additional energy input facilitating particle adsorption. ${ }^{16}$

At the highest concentrations studied (0.7 and $1 \mathrm{wt} . \%)$, the surface tension value decreases to around $50 \mathrm{mN} / \mathrm{m}$ (Figure 2). This corresponds to a surface pressure of $20 \mathrm{mN} / \mathrm{m}$, similar to that for dense successively deposited layers (Figure 1(a)).

The compression elastic modulus is given by:

$$
E=\mathrm{d} \gamma / \mathrm{d} \ln A
$$

A being the surface area. $E$ was measured (after $t \approx 10^{4} \mathrm{~s}$, when the equilibrium surface tension is reached) with oscillating bubble experiments as a function of the bulk particle concentration $c$. The results are presented in Figure 2 for particle dispersions of various bulk concentrations. The absolute value of $E$ increases markedly with increasing particle concentration $c$, reaching a value of approximately $40 \mathrm{mN} / \mathrm{m}$ at $c=0.7 \mathrm{wt} . \%$. The elastic modulus values of the layers obtained in these conditions are also similar to those of successively spread layers at $\Gamma \sim 35 \mathrm{mg} / \mathrm{m}^{2}$, just above the onset of the surface pressure increase. ${ }^{20}$

\subsection{Foam properties}

Foams can be stabilised by particles provided the contact angle between particles and water is not too low (close to $0^{\circ}$ ) or too high (close to $180^{\circ}$ ). Figure $3\left(\right.$ a) shows the samples $\left(20 \mathrm{~cm}^{3}\right.$ of aqueous dispersions of fumed silica particles of increasing hydrophobicity) after hand shaking. The volume of foam prepared with particles possessing 32 and $42 \% \mathrm{SiOH}$ is maximum and these foams were stable to coarsening and coalescence indefinitely ${ }^{3}$. The contact angle between these particles and the airwater interface is close to $90^{\circ}$, as measured on pressed discs of the same particles ${ }^{31}$ and in situ by ellipsometry ${ }^{18}$. Similar results were found with other types of particles ${ }^{12}$. When the particles are very hydrophobic and the energy input is high, an inversion to a "powder" made of liquid water droplets coated by particles (liquid "marbles") dispersed in air is seen (Figure 3(b)). ${ }^{33}$ This inversion is similar to the inversion of oil-in-water emulsions into water-in-oil emulsions, commonly observed with surfactants and particles as well. However, inversion of foams is only observed in the case of particlestabilised systems.

Particle-stabilised foams are more difficult to prepare than foams stabilised by simple surfactants. The foams cannot be produced simply by blowing air into the dispersion and procedures involving larger energy inputs are required: energetic shaking, turbulent mixing. This is probably due to the necessity to overcome the adsorption barriers described above. Similar difficulties were mentioned by Golemanov et al. in the case of emulsions. ${ }^{34}$ Tcholakova et al. discussed the problem later on by comparing the repulsive forces between a particle and a drop with the hydrodynamic forces pushing the particle toward the drop surface.$^{13}$ In the following, we will adapt their result to air bubbles. The force between a particle and the bubble surface can be written as:

$$
F \approx 2 \pi R \int_{h}^{\infty} \pi(h) d h
$$


where $R$ is the particle radius, $\pi(h)$ is the disjoining pressure of the aqueous film and $h$ is the distance between particle and bubble surfaces. In the following, we will assume that $\pi(\mathrm{h})$ is the sum of an electrostatic contribution $\pi_{\mathrm{el}}(\mathrm{h})$ and of a van der Waals contribution $\pi_{\mathrm{vdw}}(\mathrm{h})$ :

$$
\pi(\mathrm{h})=\pi_{\mathrm{el}}(\mathrm{h})+\pi_{\mathrm{vdw}}(\mathrm{h})
$$

In the case of small Debye lengths ( $\mathrm{kh}>>1), \pi_{\mathrm{el}}(\mathrm{h})$ is given by:

$$
\pi_{\mathrm{el}}(\mathrm{h}) \approx 64 \text { } \mathcal{G} \mathrm{c}_{\text {ion }} \mathrm{k}_{\mathrm{B}} T \tanh ^{2}\left(\mathrm{e} \Psi / 4 \mathrm{k}_{\mathrm{B}} \mathrm{T}\right) \exp (-\mathrm{kh})
$$

and $\pi_{\mathrm{vdw}}(\mathrm{h})$ is given by :

$$
\pi_{\mathrm{vdw}}(\mathrm{h}) \approx \mathrm{A}_{\mathrm{H}} /\left(6 \pi \mathrm{h}^{3}\right)
$$

where $A_{H}$ is the Hamaker constant (silica-water or air). The repulsive barrier between the particle and the bubble surface corresponds to the maximum force $F_{\max }$. Using $A_{H}=-10^{-20} \mathrm{~J}$, in the concentration range of the dispersions used, $F_{\max }$ is in the range $4 \times 10^{-13}-4 \times 10^{-14} \mathrm{~N}$.

The adsorption barriers exist for surfactants also, but they are much larger for particles, since their value is proportional to the particle size (see eq. 5):

Tcholakova et al. quote that the hydrodynamic force $\mathrm{F}_{\mathrm{h}}$ in laminar flow is:

$$
\mathrm{F}_{\mathrm{h}} \sim \eta \mathrm{R}^{2} \dot{\gamma}
$$

with $\eta$ being the liquid viscosity and $\dot{\gamma}$ the shear rate. In an inertial turbulent flow,

$$
F_{h} \sim \rho R^{2} S^{2 / 3} R_{b}^{2 / 3}
$$

where $\rho$ is the fluid density, $S$ the rate of energy dissipation per unit mass and $R_{b}$ the bubble radius. $S$ is equal to gradP $\mathrm{P}^{3 / 2} \mathrm{w}^{1 / 2} / \rho^{3 / 2}$ in a high pressure homogenizer, gradP being the pressure gradient and $w$ being the channel width. ${ }^{35}$ With the instrument used, ${ }^{36} \mathrm{~S} \sim 10^{5} \mathrm{~J} / \mathrm{kg} / \mathrm{s}$, and for a density $10^{3} \mathrm{~kg} / \mathrm{m}^{3}$, $F_{h} \sim 10^{-10} \mathrm{~N}$ with $R_{b} \sim 100 \mu \mathrm{m}$. The force $F_{\max }$ opposing the adsorption of the particles can therefore be overcome in the turbulent mixing system used. It can even be overcome if vigorous hand shaking is used $\left(\mathrm{S} \sim 10^{3} \mathrm{~J} / \mathrm{kg} / \mathrm{s}\right.$ and $\left.\mathrm{F} \sim 10^{-12} \mathrm{~N}\right)$, as observed. ${ }^{3,15}$

We could not make foams with the other devices at our disposal in the laboratory: bubbling gas (at most ten bubbles of millimetre radius produced per second) or in microfluidic devices (gas and liquid flow rates below $100 \mathrm{ml} / \mathrm{h}$, bubble sizes of the order of $100 \mu \mathrm{m}$ ) for which the shear rates are below $\dot{\gamma} \sim 10^{2} \mathrm{~s}^{-1}$. This leads to $\mathrm{F}_{\mathrm{h}} \sim 10^{-15} \mathrm{~N}$ for a particle of radius equal to $100 \mathrm{~nm}$, i.e. well below $\mathrm{F}_{\max }$.

It was reported that the stability of the foams produced with silica particle concentrations below 0.7 wt.\% was limited, comparable to that of the foam made with a surfactant such as sodium dodecylsulphate (SDS) ${ }^{11}$ However when the particle concentration reaches the value of $0.7 \mathrm{wt} . \%$, the stability is remarkable, with foams lasting for months. After a drainage period where the liquid drained is clear (as compared with the dispersions which are turbid), the foam evolves little with time. Studies using multiple light scattering techniques demonstrate that the average bubble radius $R_{b}$ is rather constant when for $c \geq 0.7$ wt.\% when the liquid fraction in the foam is kept constant ${ }^{11}$ (Figure 4). Visual observations after several months reveal that some of the largest bubbles are 
broken, but seemingly without influence on their neighbours. ${ }^{11}$ This suggests that coarsening is extremely slow, or even blocked. X-ray tomography of foams made by shaking reveals that some bubbles shrink, but that other bubbles become larger, suggesting that the bubbles are less well covered than in foams made by turbulent mixing and can undergo limited coarsening. ${ }^{15}$ The fact that the large bubbles are frequently broken may perhaps be related to the fracture of particle monolayers seen upon expansion, as discussed previously.

Let us try to rationalize the origin of foam stability. First, the bubbles should be well covered to resist coarsening and/or coalescence. We can estimate the surface coverage considering that the bubbles have the shape of a tetrakaidecahedron (Kelvin cell) and use the known calculations of cell volumes. ${ }^{37}$ The surface concentration is then $\Gamma=c_{f} I_{v} \phi_{b} 11.31 / 26.8$, where $c_{f}$ is the particle concentration in the foam, $I_{v}$ is the length of the vertices of hexagonal faces $\left(I_{v} \sim 0.72 R_{b}\right)$ and $\phi_{b}$ is the bubble volume fraction in the foam $\left(\phi_{b}=1-\phi_{b} \phi_{l}\right.$ being the liquid volume fraction, with $\left.c_{f}=\mathrm{c} \phi_{l}\right)$. This expression is not very different when one assumes spherical bubble geometry: $\Gamma=c_{f} R_{b} \phi_{b} / 3$. It follows that the bubble radius is $R_{b} \sim 3 \Gamma /\left(c_{f} \phi_{b}\right)=3 \Gamma /\left(c \phi_{l} \phi_{b}\right)$. If we take $\Gamma=50 \mathrm{mg} \mathrm{m}^{-2}$, a liquid volume fraction $\phi_{1} \sim 0.2$ and a particle bulk concentration of $1 \mathrm{wt} \%$, we find a radius of the order of $2.5 \mu \mathrm{m}$. Since the actual bubble radius is of the order of $25 \mu \mathrm{m}$ in the foams made by turbulent mixing, it is easy to check that the surface coverage $\Gamma$ of $50 \mathrm{mg} / \mathrm{m}^{2}$ (leading to measurable surface pressure and elasticity) can be achieved with bulk concentrations of $0.1 \mathrm{wt} . \%$. This accounts for the similarity in behaviour between SDS and particle-stabilised foams made at these low concentrations. The fact that the stability becomes almost indefinite at larger concentrations should be traced to a property other than surface coverage.

It was shown in numerical simulations by van Vliet and co-workers that coarsening (Ostwald ripening) in emulsions can be slowed down by increasing the surface elasticity. ${ }^{38}$ This was verified recently experimentally in surfactant-stabilised emulsions and foams. ${ }^{39}, 40$ When the compression elastic modulus $E$ reaches the value $E=\gamma / 2$, the coarsening stops as predicted earlier by Gibbs. Indeed, one can write the derivative of the Laplace pressure $\mathrm{P}$ with respect to bubble radius for bubbles covered by a layer with compression elastic modulus $E$ as:

$$
d P / d R_{b}=d\left(2 \gamma / R_{b}\right) / d R_{b}=-2 \gamma / R_{b}^{2}+\left(2 / R_{b}\right) d \gamma / d R_{b}=2 / R_{b}^{2}(2 E-\gamma)
$$

Since, from equation $4, E=A_{b} d \gamma / d A_{b}=1 / 2 R_{b} d \gamma / d R_{b}$ with the area of the bubble, $A_{b}=4 \pi R_{b}^{2}$.

In practice, the condition $E=\gamma / 2$ is never reached by surfactants, because coarsening is a slow process, and surfactant can desorb and adsorb freely, thus decreasing the resistance to compression (and expansion) of the layer at the bubble surfaces. Protein-stabilised foams also coarsen, although proteins may form very rigid surface layers and may not exchange with bulk (proteins are frequently irreversibly adsorbed like particles). However, these layers can slowly collapse upon increased pressure, forming multilayers. Particle layers buckle rather than collapse as seen for instance in Figure 1(b) (right). Note that it was reported recently that hydrophobins (proteins) share this property with particle layers and that they also inhibit form coarsening. ${ }^{41}$

Figure 5 shows the elastic modulus $E$ plotted as a function of the surface tension $\gamma$ in the range of particle concentrations $c$ investigated. The Figure also displays the line corresponding to the Gibbs stability criterion. Assuming that this criterion applies, only dispersions with particle concentrations 
above 0.5 wt.\% should generate foams which do not coarsen. This result is in perfect agreement with the foam stability investigations.

Note that the Gibbs argument applies to spherical bubbles. It means that if $E<\gamma / 2$, the pressure inside the bubble decreases when its radius increases, so the bubble growth is favoured. If on the contrary, $E>\gamma / 2$, the bubble can nevertheless shrink, but it can distort and adopt faceted shapes, in order to lower the Laplace pressure. This pressure may even become zero when the average curvature is zero and the driving force for coarsening vanishes. This was observed both experimentally and in simulations on armoured bubbles. ${ }^{9}$ In their simulations of single bubbles, van Vliet and co-workers also find that the coarsening stops when the surface tension become zero; ${ }^{38}$ they however assume a constant surface elastic modulus $\mathrm{E}$, which is not the case here: the surface tension saturates around $50 \mathrm{mN} / \mathrm{m}$ and never become zero even when the layer is strongly compressed.

Let us recall that a different type of bubble stabilisation can be achieved by adding salt to the particle dispersion: in this case, the silica particles form a gel in the bulk dispersion which prevents further bubble shape changes. ${ }^{42}$

The foam case is different, as it involves a collection of bubbles, some which shrink, others that grow. If the foam is isolated from the exterior (gas volume constant), coarsening stops even for finite surface tensions, provided $\mathrm{E}>\gamma / 2 .^{38}$

Note that surface viscosity appears to play an important role in the stability of surfactant foams. ${ }^{43}$ This surface viscosity originates in fact in the dissipation arising from surfactant exchanges between surface and bulk. Insoluble surfactants cannot stabilise foams, just because there is no reservoir of molecules able to cover the bubbles when created. The link between foam stability and surface viscosity is therefore likely to be indirect in this case.

Coalescence is suppressed in Pickering emulsions, provided the surface coverage reaches a large enough value $\phi_{s}{ }^{*}$. As in foams, even low coverages $\left(\phi_{s} \sim 0.2\right)$ can give rise to stable emulsions however. ${ }^{44,45}$ The interesting feature of limited coalescence can be observed: when the initial surface coverage is less than $\phi_{s}{ }^{*}$, the drops coalesce increasing the surface coverage, i.e. the total area is reduced when two drops coalesce, until their diameter reaches a value such that $\phi_{s}=\phi_{s}{ }^{*}{ }^{46}$ This feature does not appear to be observed for the relative dry foams stabilised by silica nanoparticles: the long term stability is reached above $c^{*}=0.7 \mathrm{wt} . \%$ (for foams made by turbulent mixing), $R_{b}$ is constant and equal to $R_{b}{ }^{*}$; just below this concentration, the bubble radius increases with time (Figure 4) without stabilizing at a value corresponding to the optimal surface coverage: $R_{b}$ being proportional to $\Gamma$, the limit value should be such that $R_{b}=R_{b}{ }^{*} c^{*} / c$. The origin of the differences between the foam and emulsion behaviour remains to be elucidated.

\section{Conclusions}

We have discussed investigations of the static and dynamic properties of layers of partially hydrophobic silica nanoparticles at air-water interfaces, either spread or adsorbed from aqueous dispersions. As for other micron-sized particles, the layers behave as 2-D glassy solids. The measurements strongly support the argument that the long term stability of particle-stabilised aqueous foams follows the Gibbs elasticity criterion $E>\gamma / 2$, which relates the surface tension $\gamma$ and 
the compression elastic modulus $E$ of the particle-covered interfaces. Both $\gamma$ and $E$ depend on the bulk particle concentration of the dispersion, making this therefore a key parameter for the control of foam stability.

Particle adsorption is inhibited by a pronounced energy barrier which can be overcome using strong sonication of the particle dispersions. This adsorption barrier is likely to be electrostatic in nature. Its existence also explains why the generation of stable particle foams requires high energy techniques, such as turbulent mixing. The generation of stable foams also requires suitable particles, with appropriate contact angles at the air-water surface (close to $90^{\circ}$ ).

\section{Acknowledgments}

We are grateful to Mahassine Safouane, Alfredo Cervantes, Arnaud Saint Jalmes, Wiebke Drenckhan, Duyang Zang, Bingbo Wei, Francisco Garcia Moreno and John Banhart for their contributions to the papers which are the basis of this review and for many stimulating discussions. We also thank Wacker-Chemie for the supply of particles.

\section{References}

1. S. U. Pickering, Journal of the Chemical Society, 1907, 91, 2001-2021.

2. R. G. Alargova, D. S. Warhadpande, V. N. Paunov and O. D. Velev, Langmuir, 2004, 20, 1037110374.

3. B. P. Binks and T. S. Horozov, Angewandte Chemie-International Edition, 2005, 44, 37223725.

4. U. T. Gonzenbach, A. R. Studart, E. Tervoort and L. J. Gauckler, Angewandte ChemieInternational Edition, 2006, 45, 3526-3530.

5. J. Banhart, Advanced Engineering Materials, 2006, 8, 781-794.

6. S. J. Neethling and J. J. Cilliers, Chemical Engineering Science, 2002, 57, 607-615.

7. N. D. Denkov, K. G. Marinova, C. Christova, A. Hadjiiski and P. Cooper, Langmuir, 2000, 16, 2515-2528.

8. Z. P. Du, M. P. Bilbao-Montoya, B. P. Binks, E. Dickinson, R. Ettelaie and B. S. Murray, Langmuir, 2003, 19, 3106-3108.

9. M. Abkarian, A. B. Subramaniam, S. H. Kim, R. J. Larsen, S. M. Yang and H. A. Stone, Physical Review Letters, 2007, 99.

10. A. B. Subramaniam, M. Abkarian, L. Mahadevan and H. A. Stone, Langmuir, 2006, 22, 1020410208.

11. A. C. Martinez, E. Rio, G. Delon, A. Saint-Jalmes, D. Langevin and B. P. Binks, Soft Matter, 2008, 4, 1531-1535.

12. T. N. Hunter, R. J. Pugh, G. V. Franks and G. J. Jameson, Adv. Colloid Interface Sci., 2008, 137, 57-81.

13. S. Tcholakova, N. D. Denkov and A. Lips, Physical Chemistry Chemical Physics, 2008, 10, 16081627.

14. E. Dickinson, Current Opinion in Colloid \& Interface Science, 2010, 15, 40-49.

15. A. Stocco, F. Garcia-Moreno, I. Manke, J. Banhart and D. Langevin, Soft Matter, submitted.

16. A. Stocco, W. Drenckhan, E. Rio, D. Langevin and B. P. Binks, Soft Matter, 2009, 5, 2215-2222.

17. M. Safouane, D. Langevin and B. P. Binks, Langmuir, 2007, 23, 11546-11553. 
18. D. Y. Zang, A. Stocco, D. Langevin, B. B. Wei and B. P. Binks, Physical Chemistry Chemical Physics, 2009, 11, 9522-9529.

19. D. Y. Zang, D. Langevin, B. P. Binks and B. B. Wei, Physical Review E, 2010, 81.

20. D. Y. Zang, E. Rio, D. Langevin, B. Wei and B. P. Binks, European Physical Journal E, 2010, 31, 125-134.

21. J. Nordstrom, A. Matic, J. Z. Sun, M. Forsyth and D. R. MacFarlane, Soft Matter, 6, 2293-2299.

22. P. J. Scales, F. Grieser, T. W. Healy, L. R. White and D. Y. C. Chan, Langmuir, 1992, 8, 965-974.

23. B. P. Binks and S. O. Lumsdon, Physical Chemistry Chemical Physics, 1999, 1, 3007-3016.

24. T. S. Horozov, B. P. Binks, R. Aveyard and J. H. Clint, Colloids and Surfaces a-Physicochemical and Engineering Aspects, 2006, 282, 377-386.

25. D. Vella, P. Aussillous and L. Mahadevan, Europhysics Letters, 2004, 68, 212-218.

26. P. Cicuta and D. Vella, Phys Rev Lett, 2009, 102, 138302.

27. D. Frydel, S. Dietrich and M. Oettel, Physical Review Letters, 2007, 99.

28. M. Oettel and S. Dietrich, Langmuir, 2008, 24, 1425-1441.

29. M. Oettel, personal communication

30. S. Reynaert, P. Moldenaers and J. Vermant, Physical Chemistry Chemical Physics, 2007, 9, $6463-6475$.

31. T. Kostakis, R. Ettelaie and B. S. Murray, Langmuir, 2006, 22, 1273-1280.

32. A. Bonfillon, F. Sicoli and D. Langevin, Journal of Colloid and Interface Science, 1994, 168, 497-504.

33. B. P. Binks and R. Murakami, Nature Materials, 2006, 5, 865-869.

34. K. Golemanov, S. Tcholakova, P. A. Kralchevsky, K. P. Ananthapadmanabhan and A. Lips, Langmuir, 2006, 22, 4968-4977.

35. S. Mohan and G. Narsimhan, Journal of Colloid and Interface Science, 1997, 192, 1-15.

36. A. Saint-Jalmes, M. U. Vera and D. J. Durian, European Physical Journal B, 1999, 12, 67-73.

37. L. J. Gibson and M. F. Ashby, Cellular Solids, Cambridge Univ.Press, Cambridge, UK, 1997.

38. M. B. J. Meinders and T. van Vliet, Adv. Colloid Interface Sci., 2004, 108, 119-126.

39. D. Georgieva, A. Cagna and D. Langevin, Soft Matter, 2009, 5, 2063-2071.

40. D. Georgieva, V. Schmitt, F. Leal-Calderon and D. Langevin, Langmuir, 2009, 25, 5565-5573.

41. T. B. J. Blijdenstein, P. W. N. de Groot and S. D. Stoyanov, Soft Matter, 6, 1799-1808.

42. E. Dickinson, R. Ettelaie, T. Kostakis and B. S. Murray, Langmuir, 2004, 20, 8517-8525.

43. H. Fruhner, K. D. Wantke and K. Lunkenheimer, Colloids and Surfaces a-Physicochemical and Engineering Aspects, 2000, 162, 193-202.

44. B. P. Binks, J. H. Clint, G. Mackenzie, C. Simcock and C. P. Whitby, Langmuir, 2005, 21, 81618167.

45. E. Vignati, R. Piazza and T. P. Lockhart, Langmuir, 2003, 19, 6650-6656.

46. S. Arditty, C. P. Whitby, B. P. Binks, V. Schmitt and F. Leal-Calderon, European Physical Journal $E, 2003,12,355-355$. 


\section{Figure Captions}

Figure 1. (a): Surface pressure during the first compression of spread monolayers of silica particles with $34 \% \mathrm{SiOH}$ at the air-water surface. The circles are data from successive spreading experiments in a circular trough. Solid lines correspond to data obtained in a Langmuir trough with a sensor parallel to the barriers, while dashed lines correspond to the perpendicular sensor. The differences reveal the existence of a shear modulus in addition to the compression modulus(b): Brewster angle microscopy images of the particle layers with $\Gamma=12,30$ and $60 \mathrm{mg} / \mathrm{m}^{2}$, from left to right. The scale bars correspond to $1 \mathrm{~mm}$. Above $50 \mathrm{mg} / \mathrm{m}^{2}$, the layers buckle. Data adapted from ref. 19.

Figure 2. Adsorbed monolayers of $34 \% \mathrm{SiOH}$ silica particles at the air-water surface. Surface tension $\gamma$ measured at $t=10^{4} \mathrm{~s}$ and compression elastic modulus $E$ as a function of particle concentration c. Data from ref. 17.

Figure 3. (a) Photograph of vessels containing fumed silica particle dispersions two weeks after mixing and aeration, for particles of different wettabilities. The \% SiOH content on particle surfaces (given) decreases from right to left as particles become more hydrophobic; the mixtures change from aqueous dispersions to air-in-water foams (with drained water) to water-in-air powders.

(b) Free-flowing water-in-air powder passing through a glass funnel, made with silica particles possessing $20 \% \mathrm{SiOH}$. Scale bar $=1 \mathrm{~cm}$.

(c) Air-in-water foam extruded through a serrated metal nozzle prepared with silica particles possessing 32\% $\mathrm{SiOH}$. Scale bar $=1 \mathrm{~cm}$. Data from ref. 33 .

Figure 4. Normalized average bubble radius $\left(R_{b}(0)\right.$ is the initial average radius) versus foam age for SDS- and silica particle-stabilised foams, the latter made with different bulk particle concentrations (given), prepared via turbulent mixing. The particles possess $34 \% \mathrm{SiOH}$. The initial liquid volume fraction in these foams is ca 0.25 . Arrows indicate foam stability limits and normalized average bubble radii for stable foams. Re-drawn from refs. 11 and 16.

Figure 5. Adsorbed layers of $34 \% \mathrm{SiOH}$ fumed silica particles at the air-water surface. Compression elastic modulus $E$ as a function of surface tension $\gamma$ for different concentrations. Data are from figure 2. They can be compared to the line $E=\gamma / 2$ corresponding to the Gibbs stability criterion. 
FIGURES

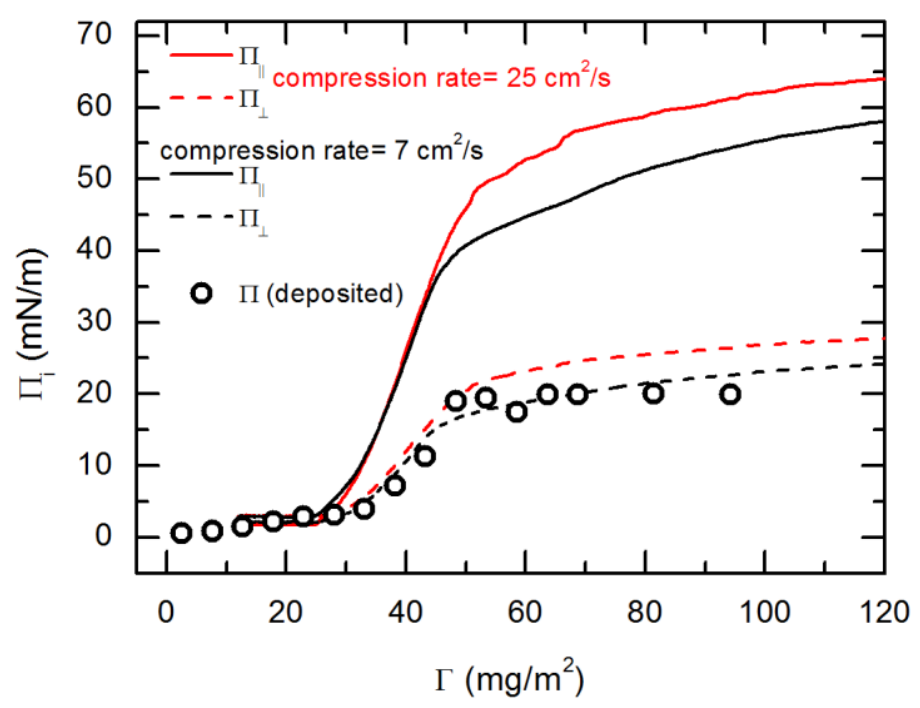

Figure 1(a)
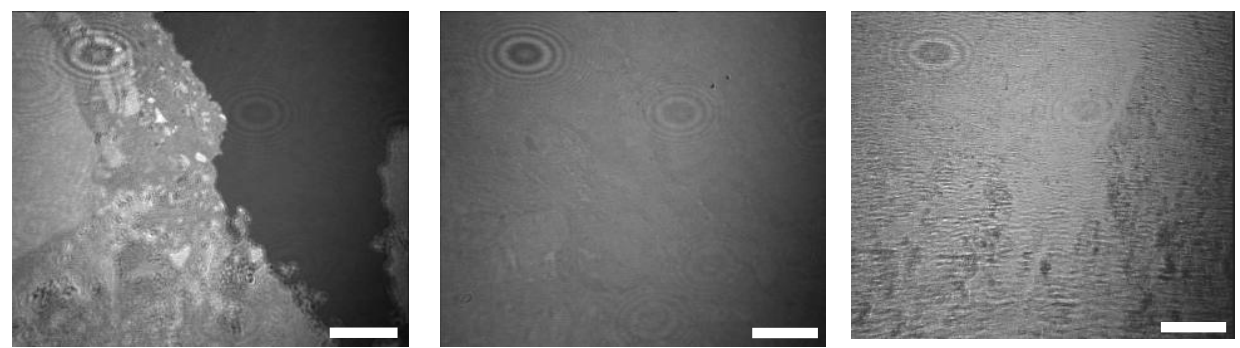

Figure 1(b)

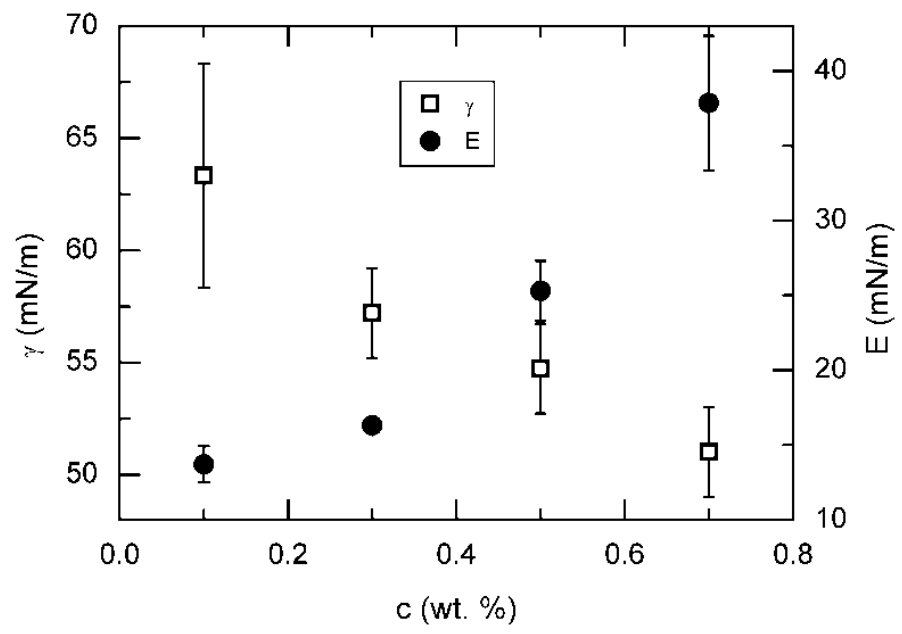

Figure 2 
(a)

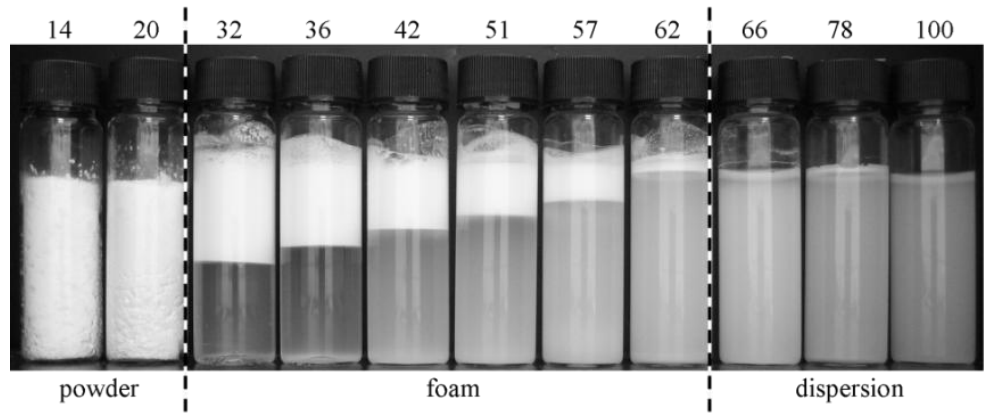

(b)

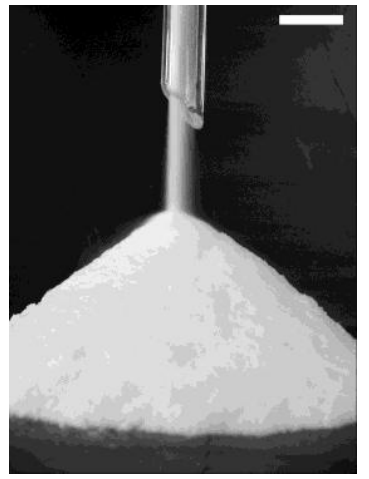

(c)

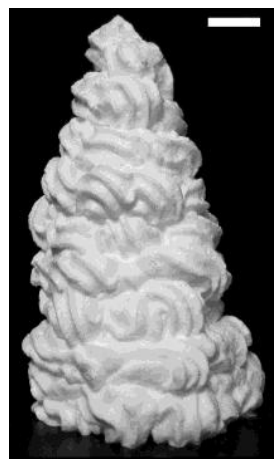

Figure 3.

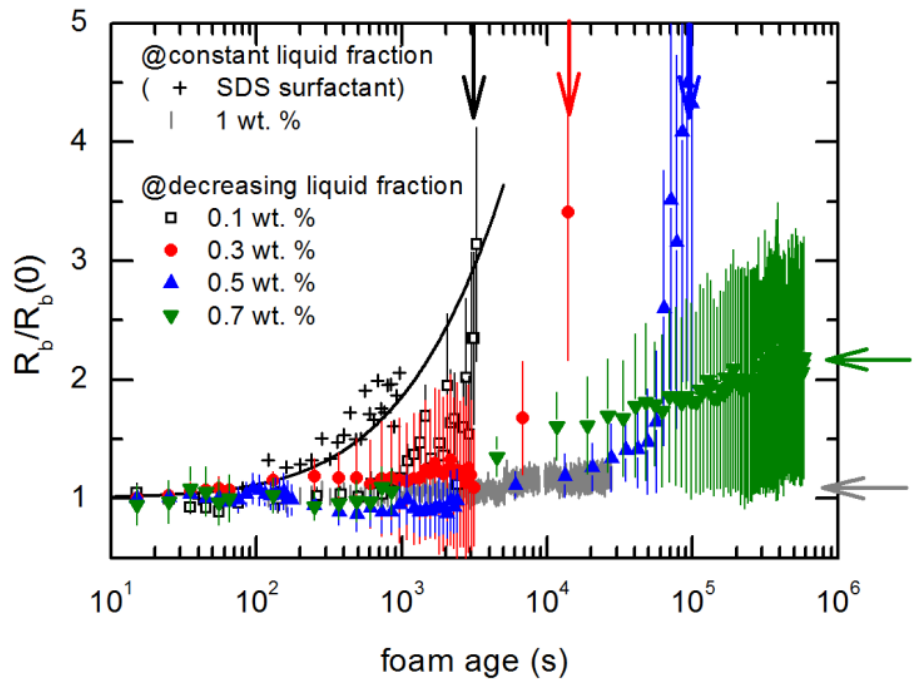

Figure 4.

Figure 5

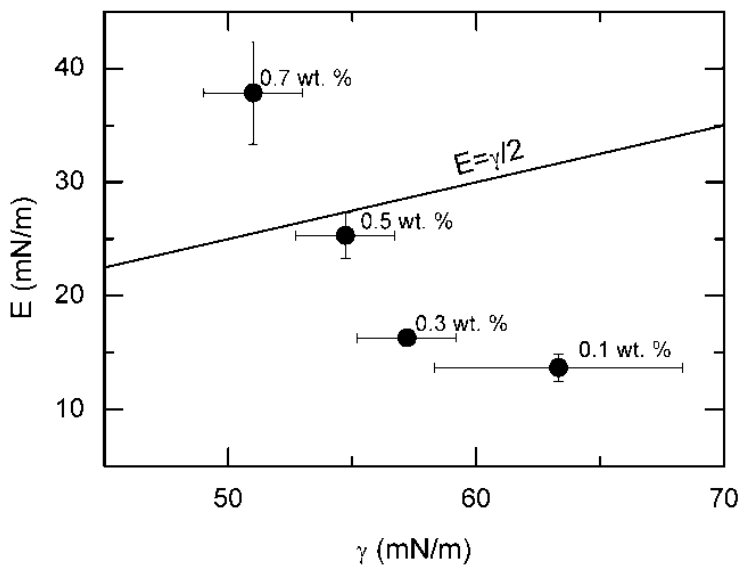

